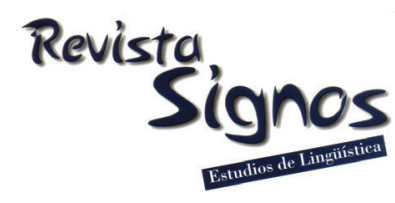

\title{
Configuraciones retórico-lingüísticas del resumen en artículos científicos de economía: Contrastes en el interior de la disciplina
}

\author{
Rhetorical-linguistic configurations of the abstract in Economics \\ research articles: Contrasts within the discipline
}

\author{
Daniela Stagnaro \\ Universidad de Buenos Aires \\ Universidad Nacional de General Sarmiento \\ ARgentinA \\ dstagnar@ungs.edu.ar
}

Recibido: 30-V-2014 / Aceptado: 30-III-2015

\section{Resumen}

En las últimas décadas, la lingüística especializada ha contribuido al conocimiento del discurso académico-científico, especialmente, de las características lingüísticoretóricas de géneros centrales de la comunicación científica. Entre ellos, el artículo científico y su resumen han recibido amplia atención por su centralidad en el proceso de investigación. Sin embargo, la bibliografía tiende a tratar el discurso de cada disciplina de manera homogénea sin considerar las ramas o los posicionamientos que se dan dentro de cada campo del saber. Inscriptos en la tradición de los estudios sobre lenguas para propósitos específicos, en este artículo, nos proponemos aportar al conocimiento de la variación intradisciplinar. Partiendo del supuesto de que existe una relación de mutua determinación entre texto y contexto, construimos un corpus de resúmenes de dos revistas especializadas representativas de enfoques opuestos dentro del campo de la economía para demostrar que estos impactan en la estructura retórica. Los resultados evidencian diferencias en las configuraciones retóricas de cada subcorpus: en un caso, 'presentación del objetivo’>‘descripción de metodología empleada'; mientras que en el otro, 'contextualización'> 'presentación del objetivo'. Así, se hacen evidentes las repercusiones de los posicionamientos epistemológicos -e ideológicos- de los autores (y las revistas) en la configuración retórica del género. Estos hallazgos contribuyen al avance del conocimiento de los lenguajes disciplinares y, a la vez, resultan relevantes para el diseño de propuestas de enseñanza que atiendan a la reflexión sobre las tensiones inherentes al campo disciplinar y a las formas retóricolingüísticas útiles a la construcción del propio posicionamiento.

Palabras Clave: Artículo de investigación, resumen, movidas retóricas, economía, enfoques o corrientes económicas. 


\begin{abstract}
Over the last few decades, Linguistics has made a number of contributions to the understanding of academic-scientific discourse, in particular of the linguistic-rhetorical features of core genres in scientific communication. Among those genres, the scientific article has received greater attention because of its centrality in the research process. However, the literature has often approached each discipline discourse homogenously, without taking into account the branches or perspectives within each field of knowledge. In the light of a tradition of studies of languages for specific purposes, this paper aims at contributing to our knowledge of intra-disciplinary variation. Based on the assumption that there is a relationship of mutual determination between text and context, we have built a corpus of abstracts from two journals which are representative of opposite approaches within the field of Economics, in order to show they have an impact on the rhetorical structure. The results show some differences between the rhetorical configurations in each subcorpus: in one case, 'stating the purpose' $>$ 'describing the methodology used'; whereas in the other, 'contextualizing' > 'stating the purpose'. Thus, it becomes evident that there is an impact of the authors' (as well as the journals') epistemological -and ideological- positioning on the rhetorical configuration of the gender. These findings contribute to the advancement of knowledge of disciplinary languages, and they are thus relevant to the design of teaching proposals that address reflection on the tensions inherent to the disciplinary field as well as on the rhetoricallinguistic forms which are useful to the construction of self-positioning.
\end{abstract}

Key Words: Research article, abstract, rhetorical moves, Economics, economic theories and approaches.

\title{
INTRODUCCIÓN
}

El pasaje de la visión positivista de la ciencia a una mirada histórica que entiende que se trata de una actividad social situada y determinada históricamente evidenció la importancia de la comunicación científica como parte de la investigación. En este sentido, el proceso de investigación finaliza con la publicación de los resultados, por lo que el artículo científico (en adelante AC) se ha constituido en el género privilegiado de este campo. Prueba de la centralidad que actualmente ocupa es su utilización cada vez más extendida a nivel internacional- como indicador para la promoción de la carrera del investigador y la obtención de financiamientos para la investigación: la cantidad de publicaciones y el impacto (es decir, la cantidad de veces que un AC es citado por otros autores es lo que se utiliza para medir el valor asignado por la comunidad a las contribuciones) se han constituido en los indicadores predilectos para el desarrollo de las políticas científicas contemporáneas (Kreimer, 2011), y de ahí la relevancia del resumen del AC en este circuito y el interés que ha concitado en diversos campos del saber que los han tomado como un objeto de estudio predilecto: la sociología de la ciencia, la historia de la ciencia, la lingüística. 
En el campo de los estudios del lenguaje científico-académico el resumen del AC o abstract ha merecido especial tratamiento, principalmente, por su brevedad, que lo hace fácilmente manipulable para los fines investigativos. En torno al español, se han desarrollado investigaciones sobre aspectos discursivos y pragmáticos (García Negroni \& Ramírez Gelbes, 2005; Beke, 2008) y acerca de las características que cada disciplina imprime al género (Bolívar, 2006). Otra línea de abordaje han sido los estudios contrastivos a partir de diversas dimensiones: diacrónica (Ciapuscio, 1998), idiomática (López Arroyo, 2000); y disciplinar (Brottier, 2000; Pérez Ruiz, 2001; da Cunha \& Sierra, 2012). Finalmente, se han realizado algunas aproximaciones a su estructura retórica (Sabaj, Toro \& Funes, 2011) o a alguno de sus componentes fundamentales, como la formulación del objetivo (González, 2011; Stagnaro, 2011) y a las relaciones léxico-semánticas que se establecen entre las distintas partes del texto: palabras clave, resumen y el cuerpo del artículo (Venegas, 2006).

La sucinta revisión bibliográfica permite verificar el incipiente avance en la construcción de conocimientos en torno al resumen del AC en español; no obstante, se observa que los estudios son mayormente de orden ejemplar y una importante dispersión temática y disciplinar. Por un lado, es evidente la necesidad de extender las investigaciones, primeramente desarrolladas para el inglés (Swales, 1990; Hyland, [2000] 2004) por su lugar hegemónico como 'lengua de la ciencia', al español. Por otro lado, se advierte que el discurso disciplinar ha sido estudiado, generalmente, como una masa homogénea: el armado de corpus de AC y de resúmenes de AC no suele atender a los diversos enfoques y/o ramas que conviven dentro de cada disciplina. Es así que no se encuentran aún abordajes sistemáticos que profundicen en los contrastes que se dan en el interior de algunas disciplinas de conformación más heterogénea del campo de ciencias sociales y humanas, como por ejemplo, la economía.

En este artículo, nos proponemos contribuir al avance del conocimiento en torno al resumen en español y los recursos del lenguaje seleccionados por la comunidad disciplinar del campo de la economía. Partimos del supuesto que establece que existen relaciones de mutua determinación entre texto y contexto (Halliday, 2001 [1978]), para explorar si las variaciones contextuales vinculadas con los posicionamientos de los autores investigadores dentro del campo disciplinar se traducen en variaciones en la selección de los segmentos funcionales que conforman el resumen del AC del dominio de la economía. A su vez, se espera que los resultados puedan nutrir el diseño de propuestas didácticas en torno a la escritura destinadas a investigadores en formación.

En función de los objetivos planteados, analizamos la estructura retórica de un corpus de resúmenes de revistas científicas del dominio de la economía que adscriben a enfoques opuestos. Así, la contribución de este trabajo se vincula con la puesta en foco de la heterogeneidad al interior de una disciplina.

A continuación, se expone brevemente el enfoque teórico en el que se inscribe 
la investigación; luego, se describe el corpus y los procedimientos seguidos; posteriormente, se informan y discuten los principales resultados: en primer lugar, en el punto 3.1., se realiza una caracterización general de los resúmenes publicados por cada una de las revistas trabajadas; en segundo lugar, en 3.2., se detallan las movidas retóricas detectadas en el corpus completo y se describen las categorías emergentes de este análisis preliminar; en tercer lugar, en 3.3. y 3.4., se describen las particularidades del subcorpus de cada una de las revistas, para, finalmente, realizar un análisis comparativo en 3.5. En el cierre del artículo, se esbozan algunas conclusiones que dialogan con los supuestos teóricos del punto de partida.

\section{Perspectiva teórica}

Esta investigación se nutre de la tradición de los análisis retórico-lingüísticos de los géneros del discurso especializado científico-académico que responde tanto al interés teórico por explicar la relación entre las formas lingüísticas y los propósitos comunicativos, es decir, entre texto y contexto, como a las preocupaciones aplicadas a la enseñanza de los géneros, particularmente, de las propuestas teórico-metodológicas que vinculan la noción de partes y segmentos textuales, de índole funcional, a rasgos léxico-gramaticales que hacen posible su identificación. Este trabajo se inscribe en esta tradición desarrollada a partir de los trabajos pioneros de Swales $(1990,2004)$ y Gnutzmann y Oldenburg (1991).

En este contexto, partimos de la noción de movida retórica (MR) de Swales:

"A "move" in genre analysis is a discoursal or rethorical unit that performs a coherent communicative function in a written or spoken discoursal. Although it has sometimes been aligned with a grammatical unit such as a sentence, utterance, or paragraph (e.g., Crookes, 1996). At one extreme, it can be realized by a clause; at the other one bay several sentences. It is a functional, not a formal, unit" (Swales, 2004: 228-229).

Entonces, entendemos que una MR es una unidad funcional que se realiza en un fragmento textual de extensión variable que ayuda al logro del propósito global del género.

Si bien este modelo para la descripción y la enseñanza de los lenguajes especiales ha sido ampliamente desarrollado para el inglés, recién en las últimas décadas, se ha comenzado a adaptar para el español. Aquí, seguimos el modelo de MR del resumen propuesto por Sabaj et al. (2011) para el español que detallamos en la Tabla 1. 
Tabla 1. Modelo de movidas retóricas para el análisis del AC en español-Resumen.

\begin{tabular}{|l|l|}
\hline 1 & Explicita el objetivo de la investigación. \\
\hline 2 & Da cuenta de los aportes de la investigación. \\
\hline 3 & Describe la metodología empleada en la investigación y/o los materiales o fuentes. \\
\hline 4 & Adelanta de forma general los resultados de la investigación. \\
\hline 5 & Interpreta los resultados. \\
\hline 6 & Da cuenta de la necesidad de investigaciones futuras. \\
\hline 7 & Hace generalizaciones. \\
\hline 8 & Revisa otras investigaciones para dar antecedentes. \\
\hline 9 & Identifica un vacío teórico en otras investigaciones. \\
\hline 10 & Identifica un vacío metodológico en otras investigaciones. \\
\hline 11 & Propone una pregunta no resuelta en otras investigaciones. \\
\hline 12 & Presenta las partes (superestructuras o temáticas) del AC. \\
\hline
\end{tabular}

Fuente: Sabaj et al. (2011: 268).

Seguramente, el lector que trabaja con el modelo Swales ya habrá advertido que la propuesta de Sabaj et al. (2011) no atiende a la distinción que este establece entre movida y paso. Cabe, entonces, realizar aquí una precisión terminológica: adscribimos a esta propuesta que los autores fundamentan en función de las dificultades empíricas de la delimitación clara entre paso y movida y a los límites más precisos del primero. En consecuencia, siguiendo a estos autores, utilizamos la denominación general 'movida' para referir a la expresión de propósitos comunicativos locales.

En este punto, cabe aclarar que teniendo en cuenta que estamos frente a un campo en construcción en relación con el español, resulta importante un esfuerzo por unificar la terminología y aunar criterios teóricos y metodológicos para lograr construir un corpus de conocimiento sistemático que permita posteriores contrastes.

\section{Estrategia metodológica}

Para explorar la variación dentro de las producciones discursivas que comunican el nuevo conocimiento en el campo de la economía, en primera instancia, hemos realizado entrevistas semiestructuradas a seis informantes clave: especialistas del campo disciplinar, todos ellos investigadores con cargos de profesor regular en el área de economía en una universidad nacional. El instrumento diseñado indaga sobre los principales enfoques, corrientes o líneas en tensión dentro del campo disciplinar y acerca de las revistas publicadas en la Argentina que representan las diversas posiciones.

Los resultados de las encuestas permitieron seleccionar dos revistas que responden a enfoques contrapuestos. Por un lado, una publicación que los especialistas reconocen como más tradicional, ortodoxa ('aunque con matices'), con tendencia más neoliberal: Revista de Economía y Estadística (en adelante REE), con una importante trayectoria, ya 
que es editada desde 1937 por el Instituto de Economía y Finanzas de la Facultad de Ciencias Económicas de la Universidad Nacional de Córdoba. Según se menciona en su página Web, se publican tanto trabajos de carácter teórico como empírico y el principal interés radica en "las investigaciones que contribuyan al conocimiento de la realidad y política económica argentina y latinoamericana, siguiendo la tradición de la publicación, con énfasis en los temas ligados al desarrollo humano"1.

Por otro lado, se escogió una revista reconocida por los especialistas como aquella que 'escapa a los enfoques tradicionales': Otra Economía. Revista Latinoamericana de Economía Social y Solidaria (en adelante, ROE). Se trata de una publicación digital de reciente aparición (2007), editada por la Red de Investigadores Latinoamericanos de Economía Social y Solidaria (RILESS). De acuerdo con la presentación disponible en la página de la revista, se propone:

"[...] difundir, debatir y contribuir a estimular la investigación crítica, teórica y empírica, sistematizando experiencias y aprendiendo de ellas, identificando y debatiendo cuestiones relevantes para los movimientos sociales y fuerzas democráticas que luchan por una economía social-mente consciente y justa, priorizando la vida de todos por sobre la acumulación de capital". ${ }^{2}$

Una vez determinadas las revistas, se construyó un corpus especializado sincrónico (Hunston, 2005) de resúmenes de AC de este dominio disciplinar. Se recolectaron todos los resúmenes de cada revista que cumplían con los siguientes criterios: publicación durante el período 2007-2010 (porque la ROE surge en este año), ubicación en la sección destinada a artículos de investigación y escritos originalmente en español ${ }^{3}$, y autores con filiación en instituciones argentinas (se descartaron los textos que indican que son traducciones). Así, se construyó una colección de veintisiete AC: trece de la REE y catorce de la ROE, aunque solo diez AC de esta última revista contienen resumen. Por lo tanto, se colectaron veintitrés resúmenes. Cabe destacar que autores como Biber (1993) plantean que el tamaño del corpus cuando se trata de textos altamente especializados no es lo más relevante. En este sentido, la finalidad de esta investigación consiste en explorar si hay variación en la estructura retórica de los resúmenes aparecidos en vías de publicación del campo económico que promueven la difusión de diferentes líneas o enfoques, es decir, no nos proponemos presentar datos estadísticos, sino explorar las opciones de la estructura retórica en relación con los diversos posicionamientos que asumen los autores dentro de un campo disciplinar. Posteriormente, los datos preliminares arrojados por este estudio se utilizarán para el análisis de un corpus más amplio que permita obtener información estadística significativa.

Esta investigación toma como objeto de estudio el resumen. Las variables analizadas son las movidas ${ }^{4}$ retóricas (en adelante MR) que se articulan dentro del resumen y los indicadores considerados son los indicios lingüísticos que señalan las diferentes funciones. 
Para el análisis retórico-lingüísitico, se realizó una aproximación top-down. En primera instancia, siguiendo la metodología del análisis de género (Swales, 2004), se llevó a cabo la identificación de las MR basada en la función del contenido del texto. Luego, se estudiaron los rasgos léxico-gramaticales recurrentes en cada una de las MR. Posteriormente, se ajustó el modelo presentado en el enfoque teórico y, así, se observaron las particularidades arrojadas por el análisis. Se cuantificaron, entonces, las ocurrencias de las distintas MR en ambos subcorpus. Finalmente, se contrastaron los resultados obtenidos del análisis de los textos de cada revista con el objetivo de hallar similitudes y divergencias e interpretarlas a la luz de la información contextual del campo disciplinar obtenida de las encuestas efectuadas a especialistas.

\section{Resultados}

\subsection{Caracterización general de los resúmenes}

La REE publicó trece artículos escritos en español desde 2005 hasta 2009. Se observa una tendencia a la autoría compartida entre dos, tres y hasta cinco investigadores. Todos los artículos publicados en esta revista en el período estudiado se encuentran encabezados por su resumen en español y su traducción al inglés. Todos ellos, salvo un caso de 2005, están seguidos de las palabras clave en las dos lenguas, excepto dos textos del mencionado año que solo las presentan en español. La extensión promedia las 150 palabras.

Las pautas para autores disponibles en la página Web de esta revista consideran el resumen y establecen precisiones en cuanto a las lenguas, la extensión y la estructura que deben tener:

Resumen: los trabajos deberán ir acompañados por un resumen en español y en inglés. El resumen debe escribirse en un solo párrafo de no más de 100 palabras. Contendrá una descripción del problema, los objetivos, la metodología y las principales observaciones y conclusiones (p. 1). ${ }^{5}$

En cuanto a los textos de la ROE, presentan una mayor variación que se evidencia en la Tabla 2 que se muestra a continuación. 
Tabla 2. Características de los resúmenes de los artículos de la ROE.

\begin{tabular}{|c|c|c|c|c|c|c|}
\hline Artículo & $\begin{array}{l}\text { Año de } \\
\text { publicación }\end{array}$ & Resumen & Idiomas & $\begin{array}{l}\text { Palabras } \\
\text { clave }\end{array}$ & \begin{tabular}{|l|} 
Cantidad \\
de autores
\end{tabular} & $\begin{array}{l}\text { Cantidad de } \\
\text { palabras }\end{array}$ \\
\hline [roe1] & 2007 & Sí & Español & No & 1 & 772 \\
\hline [roe2] & 2007 & Sí & Español & No & 1 & 306 \\
\hline [roe3] & 2007 & No & $\ldots \ldots$ & No & 2 & 0 \\
\hline [roe4] & 2007 & No & $\ldots$ & No & 1 & 0 \\
\hline [roe5] & 2008 & Sí & Español & No & 4 & 201 \\
\hline [roe6] & 2008 & Sí & Español & Sí & 2 & 155 \\
\hline [roe7] & 2008 & Sí & $\begin{array}{l}\text { Español y } \\
\text { portugués }\end{array}$ & Sí & 2 & 143 \\
\hline [roe8] & 2008 & Sí & $\begin{array}{l}\text { Español y } \\
\text { portugués }\end{array}$ & No & 1 & 151 \\
\hline [roe9] & 2008 & No & $\ldots$ & No & 1 & 0 \\
\hline [roe10] & 2008 & No & $\cdots$ & No & 1 & 0 \\
\hline [roe11] & 2009 & Sí & $\begin{array}{l}\text { Español e } \\
\text { inglés }\end{array}$ & Sí & 1 & 133 \\
\hline [roe12] & 2009 & Sí & $\begin{array}{l}\text { Español e } \\
\text { inglés }\end{array}$ & Sí & 1 & 139 \\
\hline \begin{tabular}{|l} 
[roe13] \\
\end{tabular} & 2010 & Sí & $\begin{array}{l}\text { Español e } \\
\text { inglés }\end{array}$ & Sí & 1 & 115 \\
\hline [roe14] & 2010 & Sí & $\begin{array}{l}\text { Español e } \\
\text { inglés }\end{array}$ & Sí & 1 & 155 \\
\hline
\end{tabular}

Fuente: Elaboración propia.

La Tabla 2 muestra - pese a los preceptos de construcción colectiva que se defienden desde la Economía Social y Solidaria sostenida por la revista- cierta tendencia a la autoría individual. Se observa variación en cuanto a la presencia de resumen, ya que cuatro de los artículos no tienen su resumen. Por otro lado, los resúmenes publicados durante 2007 no están seguidos de las palabras clave, sino que estas comienzan a aparecer recién a partir de los volúmenes publicados durante 2008, aunque no de manera regular. Se advierte también que, en el primer año de la revista, los resúmenes -cuando aparecen- están escritos exclusivamente en español; mientras que en 2008 se encuentran en esta lengua y seguidamente en portugués. En cambio, en 2010 los tres artículos que se publican en español están acompañados de sus resúmenes en español y en inglés, lo que pone de manifiesto un cambio en la política lingüística de la revista. Cabe destacar que cuando se presentan en dos lenguas, siempre se coloca en primer lugar el texto en español.

En lo atinente a la extensión, se pone en evidencia también cierta inestabilidad durante los dos primeros años de la publicación que comienza a estabilizarse en la publicación del segundo semestre de 2008 y permanece estable hasta la actualidad, 
entre las 115 y las 155 palabras. Ya en la convocatoria para artículos de esta revista que se llevó a cabo entre diciembre de 2009 y enero de 2010 se establecen pautas más precisas en cuanto a la extensión de los resúmenes, aunque aún no se hacen referencias relativas a la información que debe contener ni a su estructura, tal como puede observarse en el fragmento extraído de la convocatoria que figura a continuación:

"Los artículos científicos deberán estar acompañados por un resumen en idioma de origen y el Abstract de hasta 150 palabras (No se aceptará artículo sin el Abstract). Deberá estar acompañado de una mención de tres a cinco palabras clave, en ambos idiomas a los efectos de indexación bibliográfica y una búsqueda más eficiente (p. 2)"

Es interesante notar que a diferencia de la REE, que tiene disponible en su página las pautas para los autores, esta revista solo las difunde en los momentos en los que abre convocatorias para los nuevos números y suelen variar de una convocatoria a la otra.

Los datos presentados evidencian una significativa diferencia en lo que respecta a la estandarización del género propuesta desde cada revista. Por un lado, observamos que la publicación científica más tradicional con una larga trayectoria que data de 1937 presenta resúmenes con un mayor grado de estandarización en cuanto a su presencia, su extensión y las lenguas empleadas; por otro lado, en oposición, notamos la falta de regularidad en relación con estos tres aspectos en los resúmenes de la ROE de reciente aparición, por lo que esta revista podría constituir un material interesante para observar el modo como se va estandarizando el género publicación a publicación.

\subsection{Movidas retóricas del resumen de economía}

El análisis del corpus completo ha permitido detectar la realización de once MR en los resúmenes para cumplir distintas funciones retóricas: 'contextualización', 'identificación de falencias metodológicas en estudios previos', 'indicación de la necesidad de investigaciones', 'presentación del objetivo', 'señalamiento de las contribuciones del propio trabajo', 'anuncio de la hipótesis', 'descripción de los métodos empleados', 'adelanto de los resultados de la investigación', 'explicitación de las dificultades o limitaciones del trabajo', 'anticipación de las conclusiones y especificación de la superestructura del artículo'. Estos elementos funcionales se textualizan de diversas formas: mediante una o varias oraciones, o bien a través de frases con ciertos rasgos léxicogramaticales.

Los datos arrojados por el análisis señalan, por un lado, que tres de las MR propuestas por el modelo de Sabaj et al. (2011) no se presentan en nuestro corpus: 'interpreta los resultados', 'revisa otras investigaciones para dar antecedentes' y 'propone una pregunta no resuelta por otras investigaciones': movidas que involucran la polifonía y la heteroglosia, ya que ubican el propio trabajo en el concierto de 
los saberes disciplinares. Por otro lado, encontramos en el resumen movidas retóricas que en el modelo se ubican en otras secciones del AC, que también son autorreferenciales como el resumen, es decir que refieren al propio artículo/texto: 'anuncio de la hipótesis' y 'adelanto de los resultados de la investigación', que en el modelo se ubican en la introducción, y 'explicitación de las dificultades o limitaciones del trabajo', que el modelo localiza en la conclusión se encuentran en los resúmenes de economía. Finalmente, del análisis surge una categoría no contemplada en todas sus particulares por el modelo presentado por Sabaj et al. (2011). Esta MR emergente posee ciertas similitudes con las MR 'interpreta un hecho' y 'narra un hecho' halladas por Fuentes (2012) a partir del análisis de AC de historia. Propusimos la denominación 'contextualización' para ella.

Teniendo en cuenta que existe una amplia gama de bibliografía que describe las distintas MR más típicas del AC y el espacio del que disponemos en este artículo, nos concentraremos aquí exclusivamente en la descripción de la MR surgida del análisis que presenta ciertas particulares que podrían estar en relación con aspectos epistemológicos del campo disciplinar de la economía y que aún no han sido abordadas por la bibliografía.

\subsubsection{Contextualización}

Algunos de los resúmenes de ambas revistas cuentan a su inicio con un segmento que contextualiza en términos socio-históricos el objeto de estudio que se analiza a lo largo del artículo: las empresas recuperadas, los planes sociales, las ferias, los roles sociales del diseño industrial, las redes autogestivas y los intercambios alternativos. Se trata, mayormente, del relato de la transformación, el origen o el surgimiento del fenómeno que luego se analiza en el desarrollo del artículo.

Esta observación hace evidente la emergencia de una categoría no contemplada en todas sus peculiaridades en el modelo de Sabaj et al. (2011) presentado en el marco teórico. Lo hallado muestra ciertas semejanzas con la MR 'narra un hecho' descripta por Fuentes (2012) como emergente en torno a los AC de historia, no obstante, en función de la amplitud y el grado de generalidad de la categoría 'hace generalizaciones sobre el tema' podría plantearse que se trata de una forma particular de esta MR que historiza el fenómeno estudiado. Dicha historización (muy breve, por cierto) se inicia en el pasado al que se remite mediante el pretérito pluscuamperfecto, el pretérito perfecto simple y el imperfecto y llega hasta el presente como se puede notar en el ejemplo (1):

(1) A partir de la crisis de 2001 en la Argentina, numerosas ferias irrumpen como colas de feria o ferias paralelas de otras ya existentes en los parques de la Ciudad de Buenos Aires. Personas desocupadas se instalaron de manera informal y desordenada, colocando sus productos, en su mayoría objetos en desuso de sus hogares, en el piso de los parques. Con el tiempo se instalaron en puestos, $\underline{\text { renovaron }}$ 
los productos, y se organizaron institucionalmente, pero siguen siendo ferias marginales de la ciudad. Actualmente, se enfrentan a un nuevo desafío: la reconversión en ferias que comercialicen productos elaborados por los propios feriantes.

El ejemplo previo permite notar también otras características de esta MR. En primer lugar, que presenta una trama narrativa cuyas formas lingüísticas producen un efecto de objetividad, especialmente, el uso de la tercera persona de un narrador omnisciente y la inclusión de algunos elementos descriptivos de la narración, tales como, la caracterización de un hecho, su funcionalidad o sus objetivos. En segundo lugar, la prominencia de las circunstancias temporales en posición temática que organizan la narración, puesto que sirven, justamente, para situar y ubicar temporalmente el objeto de estudio, lo que construye discursivamente una dimensión histórica. En tercer lugar, se advierte en interior de esta MR el establecimiento de relaciones lógicas, principalmente, las que indican causas y consecuencias de los hechos relatados, como se puede ver en el ejemplo (2):

(2) Las denominadas "empresas recuperadas" se han establecido en la Argentina a partir de un proceso de crisis que culminó con el abandono y la quiebra de organizaciones, que no pudieron salir de la difícil situación económica-financiera por sus propios medios. Esta circunstancia, afectó a sus trabajadores que avizoraban la desaparición gradual de sus fuentes de trabajo.

Finalmente, en lo atinente a la ubicación de esta MR, el 100\% de los casos hallados en el corpus se ubican en posición inicial, esto es, abren el resumen, por lo que se solapa aquí también la función de presentar el tema de estudio en perspectiva histórica en función de las características descriptas previamente. Esta selección de los autores presenta el objeto de estudio a partir de determinadas configuraciones sociales, históricas, políticas y económicas. Este anclaje en la dinámica histórica se distancia del abordaje de objetos económicos abstractos y ahistóricos. Más adelante mostraremos que la elección de esta MR por parte de los autores se vincula con los posicionamientos epistemológicos -e ideológicos- (Bourdieu, [1976] 2001) de las revistas y los autores investigadores.

\subsection{El resumen de la Revista de Economía y Estadística}

El análisis muestra una tendencia general en los resúmenes de la REE a la configuración retórica en dos o tres MR, solo uno de ellos presenta cinco segmentos y otro, cuatro. Se identificó un total de nueve movidas en este primer grupo: 'presentación del objetivo', 'descripción de metodología empleada', 'identificación de falencias en estudios previos', 'adelanto de los resultados de la investigación', 'anticipación de las conclusiones', 'especificación de la superestructura del artículo', 'contextualización', 'señalamiento de las contribuciones del trabajo' y 'explicitación de las dificultades o limitaciones del trabajo’. A su vez, no se ha registrado ninguna 
ocurrencia de dos de las movidas caracterizadas en el apartado previo: 'indicación de la necesidad de investigaciones' y 'anuncio de la hipótesis'. La Tabla 3 muestra el porcentaje de ocurrencia de cada uno de los movimientos mencionados.

Tabla 3. Movimientos del resumen en la REE.

\begin{tabular}{|l|l|}
\hline Movimientos retóricos & REE \\
\hline Presentación del objetivo & $100 \%$ \\
\hline Descripción de metodología empleada & $70 \%$ \\
\hline Identificación de falencias en estudios previos & $20 \%$ \\
\hline Adelanto de los resultados de la investigación & $20 \%$ \\
\hline Anticipación de las conclusiones & $20 \%$ \\
\hline Especificación de la superestructura del artículo & $20 \%$ \\
\hline Contextualización & $10 \%$ \\
\hline Señalamiento de las contribuciones del trabajo & $10 \%$ \\
\hline Explicitación de las dificultades o limitaciones del trabajo & $10 \%$ \\
\hline
\end{tabular}

Fuente: Elaboración propia.

Los datos relevados en la Tabla 3 evidencian una mayor frecuencia de dos MR presentan. En primer lugar, la totalidad de los resúmenes de este subcorpus presenta el objetivo del artículo o de la investigación que se informa. Cabe señalar que en muchos casos, en esta MR se establece entre la investigación, el artículo y los autores investigadores una relación metonímica. En segunda instancia, es sumamente significativa la ocurrencia de la MR en la que se da cuenta de la metodología mediante la cual se obtuvieron los datos y los resultados. En esta revista parece haber un especial interés por este aspecto intrínseco a la investigación, ya que, tal como muestra la Tabla 3, el 70\% de los textos se ocupa de explicitar los métodos o los procedimientos utilizados. Cabe notar que no se trata de la mera mención de tipos o categorías de una clasificación de métodos (cualitativa, cuantitativa, etc.), sino que los autores presentan información con mayor grado de detalle.

En función de los datos porcentuales de la ocurrencia de cada MR, no sorprende, entonces, que la tendencia detectada con respecto a la configuración retórica consista en una estructura con dos componentes: 'presentación del objetivo' > 'descripción de metodología empleada'. En algunos resúmenes, se suma a continuación de esta estructura alguno de los siguientes componentes retóricos: 'adelanto de los resultados de la investigación', 'anticipación de las conclusiones', 'especificación de la superestructura del artículo', 'señalamiento de las contribuciones del trabajo' y/o 'explicitación de las dificultades o limitaciones del trabajo'. Así, si bien las movidas iniciales son fijas, en las finales se advierte cierta flexibilidad en las selecciones realizadas por los autores. 


\subsection{El resumen de la Revista Otra Economía}

El subcorpus de los resúmenes de la ROE presenta menor variedad de MR que el grupo anterior. Mientras que de los resúmenes de la REE obtuvimos once MR, en este grupo solo se identificaron siete: 'presentación del objetivo', 'contextualización', 'descripción de metodología empleada', 'indicación de la necesidad de investigaciones', 'anuncio de la hipótesis', 'anticipación de las conclusiones' y 'especificación de la superestructura del artículo'. Se advierte, en consecuencia, que cuatro de las MR que aparecen en los textos de la REE aquí no registran ninguna ocurrencia: 'identificación de falencias en estudios previos', 'señalamiento de las contribuciones del trabajo', 'adelanto de los resultados de la investigación' y 'explicitación de las dificultades o limitaciones del trabajo'.

La Tabla 4 registra el detalle completo de las MR halladas y el porcentaje de ocurrencia de cada una de ellas en el subcorpus de la ROE.

Tabla 4. Movimientos del resumen en la ROE.

\begin{tabular}{|l|l|}
\hline Movimientos retóricos & ROE \\
\hline Presentación del objetivo & $100 \%$ \\
\hline Contextualización & $70 \%$ \\
\hline Descripción de metodología empleada & $20 \%$ \\
\hline Indicación de la necesidad de investigaciones & $10 \%$ \\
\hline Anuncio de la hipótesis & $10 \%$ \\
\hline Anticipación de las conclusiones & $10 \%$ \\
\hline Especificación de la superestructura del artículo & $10 \%$ \\
\hline
\end{tabular}

Fuente: Elaboración propia.

Los resúmenes de la ROE están constituidos por dos o tres componentes, sin excepciones. Los datos presentados en la Tabla 4 permiten advertir que la MR 'presentación del objetivo' se registra en todos los textos y que la contextualización social, histórica y/o política del fenómeno que se estudia se encuentra en el 70\% de estos. Estos porcentajes se relacionan con la tendencia predominante en la configuración retórica de este subcorpus: 'contextualización' > 'presentación del objetivo'. En algunos casos esta estructura se continúa con una tercera MR que varía entre las siguientes: 'anuncio de la hipótesis' o 'descripción de metodología empleada'. En un resumen, se intercala la MR 'indicación de la necesidad de investigaciones' entre la 'contextualización' y la 'presentación del objetivo'.

Cabe señalar que se detecta solo un caso que concuerda con la configuración preponderante en los resúmenes de la REE: 'presentación del objetivo' > 'descripción de metodología empleada'. 


\subsection{Resonancia de los posicionamientos epistemológicos en las realizaciones retóricas del resumen}

Si contrastamos los datos obtenidos con respecto a las configuraciones retóricas de los resúmenes de las dos revistas, advertimos que cada subcorpus presenta particularidades específicas. En el Gráfico 1 se comparan los resultados de ambos grupos de textos.

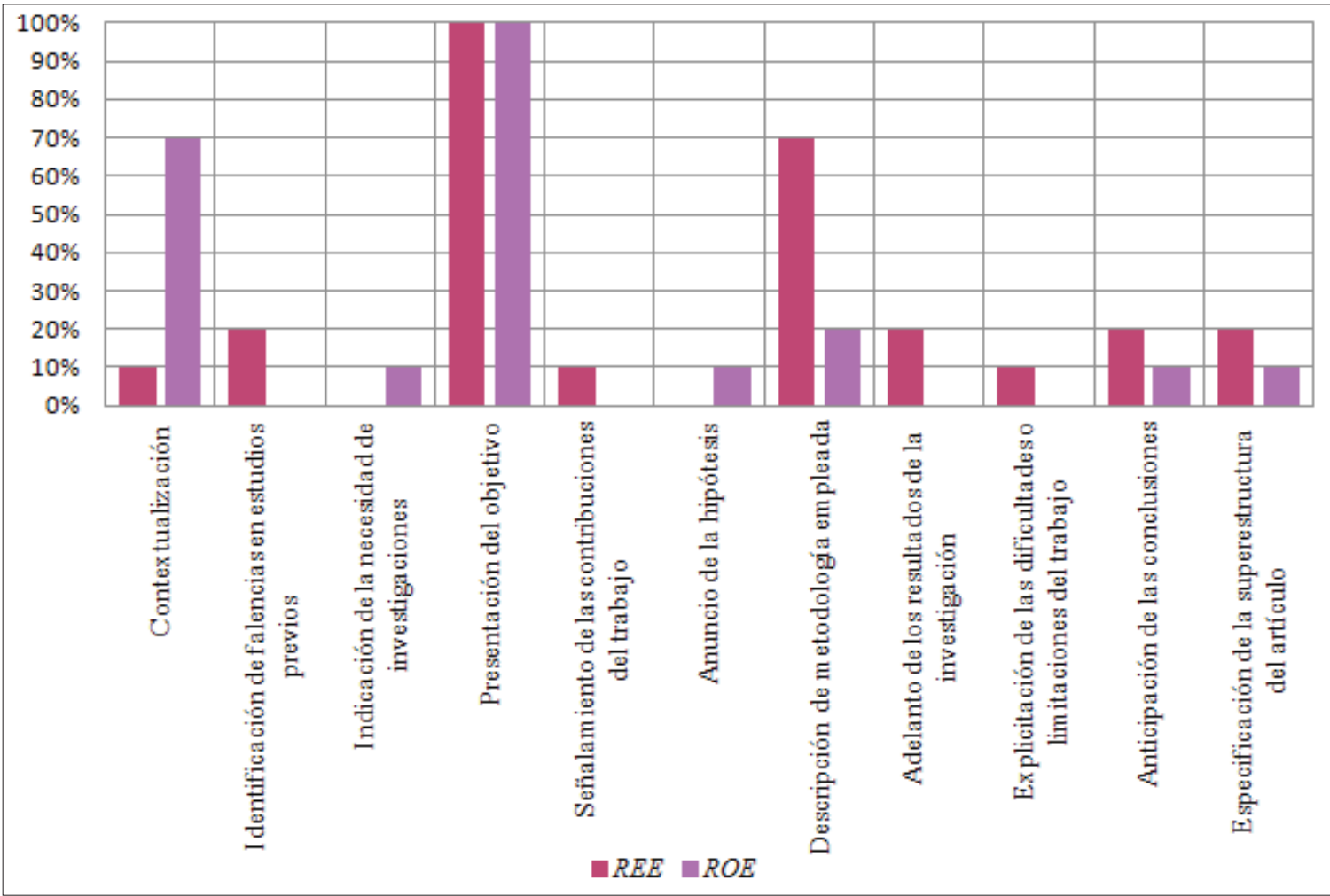

Gráfico 1. Comparación de ocurrencias de MR en los resúmenes de la REE y la ROE.

El Gráfico 1, por un lado, visibiliza las coincidencias en las MR que presentan ambos subcorpus, como la recurrencia de la MR 'presentación del objetivo', que la bibliografía sobre el resumen señala como obligatoria (por ejemplo, Samraj, 2005; Pho, 2008), en la totalidad del corpus. Sin embargo, este dato muestra ciertas divergencias con lo hallado en torno al resumen de otras disciplinas, por ejemplo, Fuentes (2012) encuentra que en el caso de su corpus de resúmenes de historia en español solo el 66,6\% presenta esta MR.

Por otro lado, el Gráfico 1 pone de relieve algunas diferencias entre los resúmenes de las dos revistas: en primer lugar, se observa una mayor variedad de MR en los resúmenes de la REE; en segundo lugar, se advierte la preponderancia de distintas 
MR en cada uno de los grupos: mientras que en la REE predomina la MR 'descripción de metodología empleada', en la ROE sobresale la MR ‘contextualización’. A su vez, la MR prominente en cada uno de los subcorpus registra un porcentaje de ocurrencia muy bajo en relación con lo arrojado por el otro subcorpus: 'contextualización' aparece en el 70\% de los resúmenes de la ROE, pero solo en el 10\% de la REE; mientras que 'descripción de metodología empleada' se encuentra en el 70\% de los resúmenes de la REE, pero solo en el $20 \%$ de la ROE.

La escasa aparición de la MR 'descripción de metodología empleada' al igual que el amplio porcentaje de ocurrencia de la MR 'contextualización' en los resúmenes de la ROE evidencian una cercanía entre estos resúmenes y los de historia estudiados por Fuentes (2012), quien registra solo un $8 \%$ de la primera MR y señala la emergencia de una MR con trama narrativa que se asemeja a la que hemos denominado 'contextualización'. En cambio, los resúmenes de la REE tienen una mayor cercanía con los hallazgos realizados por los estudiosos de textos provenientes de las ciencias fácticas, como por ejemplo, la medicina (Ciapuscio \& Otañi, 2002), al poner el énfasis en los métodos.

Si cruzamos los datos obtenidos con las respuestas de los especialistas recogidas en las encuestas, se enriquece la interpretación de las relaciones entre texto y contexto. Frente a la pregunta acerca de cuáles son las principales posturas/corrientes/líneas del campo de la economía en la actualidad, uno de los especialistas establece la siguiente distinción que podría resultar pertinente para comprender las preferencias por ciertas MR en los resúmenes de cada revista:

"Tradicionalmente, yo contestaría que existen dos grandes corrientes del pensamiento económico: uno, ortodoxo (neoclásico, de economía "pura", a-histórico, con pretensión de universalidad); otro, heterodoxo (dinámico, histórico, en el que entrarían desde el marxismo hasta el estructuralismo pasando por el regulacionismo, los neo-schumpeterianos, etc.). Mi impresión es que ese esquema está dejando de estar vigente y que hay formas muy novedosas de ortodoxia y heterodoxia pero que aún no llegan a definir un panorama tan claro como en el pasado. Me parece que es un momento de transición (sic) [E1]".

La distinción que este economista establece entre la economía "pura" y a-histórica y el interés por la dinámica histórica podría arrojar luz sobre las selecciones retóricas preponderantes en cada revista. La tendencia de los resúmenes de la REE a la estructura 'presentación del objetivo' > 'descripción de metodología empleada' podría estar en relación con los posicionamientos de los autores, en términos muy generales, dentro de la primera de las vertientes que representa una visión universalista y a-histórica, y que varios de los especialistas encuestados identificaron con el neoliberalismo. En cambio, la tendencia predominante de los resúmenes de la ROE a la configuración retórica 'contextualización' > 'presentación del objetivo' podría estar señalando el 
posicionamiento de los autores investigadores, en términos muy generales, dentro de la segunda vertiente que atiende a la dinámica histórica.

Estas observaciones dialogan, por un lado, con el estudio contrastivo del resumen y la introducción de dos disciplinas (Wildlife Behavior and Conservation Biology) realizado por Samraj (2005). Allí, encuentra que la variación disciplinar se manifiesta en la estructura genérica. Por otro, con lo hallado por Vallejos Llobet y García Zamora (2006) en su estudio diacrónico en torno a las repercusiones de los cambios de paradigmas epistemológicos en las convenciones discursivas de los géneros utilizados por una comunidad. Nuestros hallazgos permiten extender estas observaciones en torno a la variación al interior de un campo disciplinar en un mismo momento para postular que los diferentes posicionamientos epistemológicos que conviven en tensión dentro de un mismo campo disciplinar también repercuten en las selecciones retóricas de los investigadores-escritores e imprimen rasgos particulares al género AC. De esta forma, se refuerza la relación de los géneros con la actividad social y su heterogeneidad y dinamismo en función de los diversos objetivos de los productores.

\section{CONCLUSIONES}

Recapitulando, hemos iniciado este artículo planteando el supuesto hallidayano de la existencia de una relación dialéctica entre texto y contexto y nos hemos propuesto llevar a cabo una contrastación empírica, ya que si bien se ha presentado evidencia de las variaciones retórico-lingüísticas entre lenguas, culturas y disciplinas, aún no se ha aportado información suficiente en torno a las variaciones intradisciplinarias.

Hemos analizado las configuraciones retóricas de los resúmenes de dos revistas especializadas que responden de alguna manera a posicionamientos epistemológicos e ideológicos históricamente antagónicos dentro del campo de la economía: la ortodoxia y la heterodoxia. El análisis de los resúmenes de ambas revistas ha mostrado que si bien los textos comparten ciertas MR, en los resúmenes de cada revista predomina una configuración particular: se ha advertido que en la REE la tendencia general es la estructuración a partir de dos movimientos, 'presentación del objetivo' > 'descripción de metodología empleada'; mientras que en la ROE es 'contextualización' > 'presentación del objetivo'. Hemos vinculado estos hallazgos con los datos obtenidos de las encuestas para mostrar que estas dos tendencias podrían estar determinadas por los posicionamientos epistemológicos e ideológicos en disputa dentro del campo disciplinar. En el caso de los resúmenes de la REE, llevar a cabo la presentación del propio trabajo mediante la explicitación del objetivo del texto o de la investigación y de los instrumentos, métodos y procedimientos denota un interés preponderante por aspectos metodológicos de la tarea científica que excluyen, generalmente, la dimensión social, política e histórica del objeto de estudio y la ciencia económica. En contraste, en el caso de los resúmenes de la ROE se opta por presentar el texto y el tema partiendo de la historización de este último o del objeto de estudio, enfatizando, 
de este modo, tanto la dimensión social como la política con las que interactúa la economía social y solidaria que prescribe esta revista con propósitos de intervenir sobre la realidad. En este sentido, realizar una contextualización respondería a la necesidad de distanciarse de una idea de universalidad de la economía para considerar, en cambio, la construcción socio-histórica de los objetos de estudio de la economía.

En consecuencia, las variaciones detectadas en las MR seleccionadas en los resúmenes de revistas especializadas del dominio de la economía aportan evidencia acerca de la hipótesis de que textos con igual grado de especialidad y función, pero con diferentes perspectivas en el abordaje del objeto dentro del campo de una misma ciencia presentan variaciones retóricas, por lo que sería necesario incorporar este parámetro para el análisis de los géneros científicos. Este hallazgo representa un importante aporte a los desarrollos de los estudios lingüísticos y requiere el avance sobre mayores porciones de discurso que permitan realizar precisiones y especificaciones de los factores determinantes dentro de cada contexto disciplinar. Es decir, esta primera aproximación señala algunas tendencias en relación con la variación intradisciplinar que requiere corroborarse en corpus más amplios que permitan obtener información estadística significativa.

Por último, estas observaciones también revisten especial interés para el campo de la enseñanza de los lenguajes especializados, en tanto ponen de relieve el carácter social e histórico de los géneros y de la ciencia y, por lo tanto, la necesidad de incluir en las clases y en los materiales didácticos reflexiones sobre las tensiones que se dan dentro de cada campo disciplinar y las formas retórico-lingüísticas que sirven para la construcción del propio posicionamiento dentro del campo al que se pretende acceder. 


\section{REFERENCIAS BIBLIOGRÁFICAS}

Beke, R. (2008). El discurso académico: La atribución del conocimiento en la investigación educativa. Núcleo, 25, 13-35.

Biber, D. (1993). Representativeness in corpus design. Literary and linguistic Computing. 8(4), 243-256.

Bolivar, A. (2006). La función de la evaluación en artículos y ensayos humanísticos. En J. Falk, J. Gille \& F. Wachtmeister Bermúdez (Eds.), Discurso, interacción e identidad (pp. 111-136). Estocolmo: Stockholms Universitet.

Bourdieu, P. ([1976] 2001). El campo científico. Redes: Revista de Estudios Sociales de la Ciencia, 1, 131-159.

Brottier, O. (2000). El español con propósitos específicos: El resumen (abstract) del artículo científico. En M. Martín Zorraquin \& C. Díez Pelegrín (Eds.), Actas del XI Congreso Internacional de ÁSELE. Zaragoza.

Ciapuscio, G. (1998). Los resúmenes de la revista Medicina: Un enfoque diacrónicocontrastivo. Signo y Seña, 10, 217-240.

Ciapuscio, G. \& Otañi, I. (2002). Las conclusiones de los artículos de investigación desde una perspectiva contrastiva alemán-español. RILL, 15, 117-133.

da Cunha, I. \& Sierra, G. (2012). Hacia la búsqueda de rasgos discursivos específicos de dominios especializados. En Actas del XII Simposio de RITerm La terminología: puente ineludible de una sólida mediación cultural, Buenos Aires, Argentina.

Fuentes, M. (2012). El discurso científico de la historia: Análisis estructural y retórico de los artículos de investigación en historia. Boletín de Filología, XLVII (1), 89-110.

García Negroni, M. M. \& Ramírez Gelbes, S. (2005). Polémica y agente. El caso de sin embargo y no obstante en los abstracts académicos. En S. Santos \& J. Panesi (Eds.), Actas del Congreso Internacional: Debates actuales. Las teorías criticas de la literatura y la lingüística. FFyL, Universidad de Buenos Aires. Buenos Aires, Argentina.

Gnutzmann, C. \& Oldenburg, H. (1991). Contrastive text linguistics in LSP-Research: Theoretical considerations and some peliminary findings. En H. Schröder (Ed.), Subject-oriented Texts: Language for Special Purposes and Text Tehorie (pp. 101136). Nueva York/ Berlín: de Gruyter. 
González, C. (2011). La formulación de los objetivos en artículos de investigación científica en cuatro disciplinas: Historia, lingüística, literatura y biología. Revista Linguagem em (Dis)curso, 11(2), 401-429.

Halliday, M. ([1978] 2001). El lenguaje como semiótica social. Buenos Aires: FCE.

Hunston, S. (2005). Corpora in applied Linguistics. Cambridge: Cambridge University.

Hyland, K. ([2000] 2004). Disciplinary Discourses. Social Interactions in Academic Writing. Michigan, USA: The University of Michigan Press.

Kreimer, P. (2011). La evaluación de la actividad científica: Desde la indagación sociológica a la burocratización. Dilemas actuales. Propuesta Educativa, 20(3), 59-77.

López Arroyo, B. (2000). Estudio descriptivo comparado inglés/español de la representación del conocimiento en los abstracts de las ciencias de la salud. Tesis doctoral, Universidad de Valladolid, Valladolid, España [en línea]. Disponible en: http://www. cervantesvirtual.com/buscador $/$ ?q $=1 \% \mathrm{C} 3 \% \mathrm{~B} 3$ pez + arroyo\#posicion

Pérez Ruiz, L. (2001). Análisis retórico contrastivo: El resumen lingüístico y médico en inglés y español. Tesis doctoral, Universidad de Valladolid, Valladolid, España [en línea]. Disponible en: http://www.cervantesvirtual.com/buscador/?q=P\%C 3\%A9rez+Ruiz\#posicion

Pho, P. (2008). Research article abstracts in applied linguistics and educational technology: A study of linguistic realizations of rhetorical structure and authorial stance. Discourse Studies, 10, 231-250.

Sabaj, O., Toro, P. \& Fuentes, M. (2011). Construcción de un modelo de movidas retóricas para el análisis de artículos de investigación del español. Onomázein, 24, 245-271.

Samraj, B. (2005). An exploration of a genre set: Research article abstracts and introductions in two disciplines. English for Specific Purposes, 24, 141-156.

Stagnaro, D. (2011). Variantes del artículo de investigación en el dominio de la economía: Exploración de las configuraciones de la transitividad. Texturas. Estudios Interdisciplinarios sobre el Discurso, 11, 111-136.

Swales, J. (1990). Genre analysis: English in academic and research settings. Glasgow: Cambridge University Press.

Swales, J. (2004). Research genres: Explorations and applications. Cambridge: Cambridge University Press. 
Vallejos Llobet, P. \& García Zamora, M. (2006). Lo epistemológico en lo retórico: Una mirada al discurso de las ciencias de la educación desde la LSF. Actas del $33^{\circ}$ Congreso Internacional de Lingüística Sistémico Funcional.

Venegas, R. (2006). La similitud léxico-semántica en artículos de investigación científica en español: Una aproximación desde el Análisis Semántico Latente. Revista Signos. Estudios de Lingüística, (39)60, 75-106 [en línea]. Disponible en: http://www.scielo.cl/scielo.php?script=sci_arttext\&pid=S07180934200600 0100004\&lng $=$ es\&nrm=iso

\section{NOTAS}

1 http://ief.eco.unc.edu.ar/index.php?option $=$ com_content\&view $=$ article\&id $=148 \&$ Itemid $=77$ El resaltado corresponde al original.

2 http://www.riless.org/otraeconomia/sobre\%20la\%20revista.html

3 Este requisito responde a que este trabajo se enmarca en el proyecto PICT "Variedades del español de la Argentina: estudios textuales, gramaticales y léxicos" que se propone contribuir a la descripción de la variedad especializada del español de la Argentina.

4 Tomamos como unidad de análisis las porciones textuales mínimas que cumplen una función retórica. Las especificaciones en cuanto a las movidas y los pasos no suelen ser del todo claras y distintos autores utilizan las diferentes denominaciones para nombrar un mismo segmento. A los fines de nuestro estudio, interesa aclarar que, tal como señalamos en la sección perspectiva teórica, analizamos las unidades funcionales más pequeñas siguiendo a Sabaj et. al 2011: 'presentación del objetivo', 'descripción de metodología empleada', 'identificación de falencias en estudios previos'.

5 http://ief.eco.unc.edu.ar/index.php?option $=$ com_content\&view $=$ article\&id=146\&Itemid $=24$

6 www.riless.org/otraeconomia/convocatoria.html

7 Los resaltados son nuestros. 